\title{
Performance Test of a TMS Calorimeter
}

\author{
ل. Engler, H. Keim, B. Wild \\ : Institut für Kemphysik
}

\section{Kernforschungszentrum Karlsruhe}


KERNFORSCHUNGSZENTRUM KARLSRUHE

Institut für Kernphysik

KfK 4085

Performance Test of a THS Calorineter

J. Engler, H. Keim and B. Wild

Kernforschungszentrum Karlsruhe GmbH, Karlsruhe 


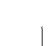


ABSTRACT

Performance tests of a first calorimeter module using the room temperature liquid tetramethylsilane (TMS) as active element are described. Normal carbon steel has been used as absorber. The charge yield is $70 \%$ of that in a very pure sample of the liquid. A long term stability of the signal with a lifetime of half a year has been realized.

BETRIEB EINES TMS KALORIMETERS

\section{ZUSAMMENFASSUNG}

Es werden Ergebnisse über den Betrieb eines ersten Kalorimeters gegeben, das die Flüssigkeit Tetramethylsilan (TMS) als aktives Element benutzt. Als Absorber wurde normaler Kohlenstoffstahl verwendet. Die Ladungsausbeute betrug 70\% derjenigen, der in einer sehr reinen Probe der Flüssigkeit erhaltenen Ausbeute. Als Langzeitstabilität wurde eine Lebensdauer des Signals von einem halben Jahr gemessen.

Submitted to Nuclear Instruments and Methods 


\section{Introduction}

Sampling calorimeters have gained more and more importance during the last decade for high energy particle detection.

Experience with these devices has shown that liquid ionization chambers as active elements between the inert absorber plates offer many advantages especially with regard to long term performancel. Up to now, only cryogenic liquids have been used, and in calorimetry only liquid argon. Several large scale detectors currently under construction have chosen this technique.

Nevertheless, the use of a cryogenic liquid also implies drawbacks. Apart from the laborious work of maintaining a cryogenic system, the necessity of a cryostat complicates the lay-out of a detector. A hermetic $4 \pi$ detector without holes and cracks is difficult to realize. With room temperature liquids, these disadvantages can be avoided. Besides this, large calorimeters could be built in conveniently small modules, which are grouped together to form a large detector assembly.

A further advantage concerns hadron calorimetry. Nearly all candidates for room temperature liquids contain large amounts of hydrogen. In conjunction with uranium as absorber material this can compensate the binding energy losses of nuclear break-up due to induced fission ${ }^{2}$. It has, however, to be proven that the charge collection for the low energy recoiling protons from fission neutrons is not too saturated to allow an accurate energy measurement.

For our test, we chose tetramethylsilane TMS $\left.=\mathrm{Si}_{(\mathrm{CH}}\right)_{4^{\circ}}$. It is one of the best warm liquids investigated so far ${ }^{3}$. It has a high charge yield and the fastest drift velocity. The drift time for $1 \mathrm{~mm}$ gap distance is $50 \mathrm{~ns}$ at an electric field of $20 \mathrm{kV} / \mathrm{cm}$ and $30 \mathrm{~ns}$ at $30 \mathrm{kV} / \mathrm{cm}$. The liquid is commercially available at a reasonable price. Its vapour pressure is 0.75 bar at $20^{\circ} \mathrm{C}$ and its boiling point is at $26.5^{\circ} \mathrm{C}$. 
For the absorber material, we chose ordinary carbon steel plates. We intended in this test to prove that even the return flux iron in large detectors could be instrumented this way. The complete iron masses would be built up out of many individual modules which could be operated independently of each other.

\section{Calorimeter Module}

The lay-out of the module is sketched in Fig. 1. It consisted of 16 steel plates, each $15 \mathrm{~mm}$ thick with a planed surface. No special treatment like galvanizing was adopted in order to keep the fabrication cost low. The TMS gaps were $6 \mathrm{~mm}$ wide. In the middle of the gaps pick-up boards were inserted which were held by teflon washers between the steel plates. These boards were made from two layers of copper clad polyimid foils, each $125 \mathrm{\mu m}$ thick, glued together. On the two outer sides, a pad structure of about $8 \times 8 \mathrm{~cm}^{2}$ carried the high voltage. The signals were coupled out through the polyimid to a nearly identical structure of pads of a slightly smaller size which were etched on the inner side of one of the polyimid foils, and connected to the preamplifier.

Longitudinally, in beam direction, 8 pads were ganged together defining one tower. The bus connection was realized with $0.6 \mathrm{~mm}$ ceramic plates, onto which the working resistors of the voltage feeds had been printed. In total, the calorimeter was read out via 60 amplifier channels.

A photograph of the calorimeter is shown in Fig. 2. The whole structure was mounted on a thick ground plate and enclosed by a $3 \mathrm{~mm}$ thick cover of stainless steel. The preamplifiers were located immediately on top of the calorimeter in a copper box and connected by standard glass feed-throughs to the bus planes. 


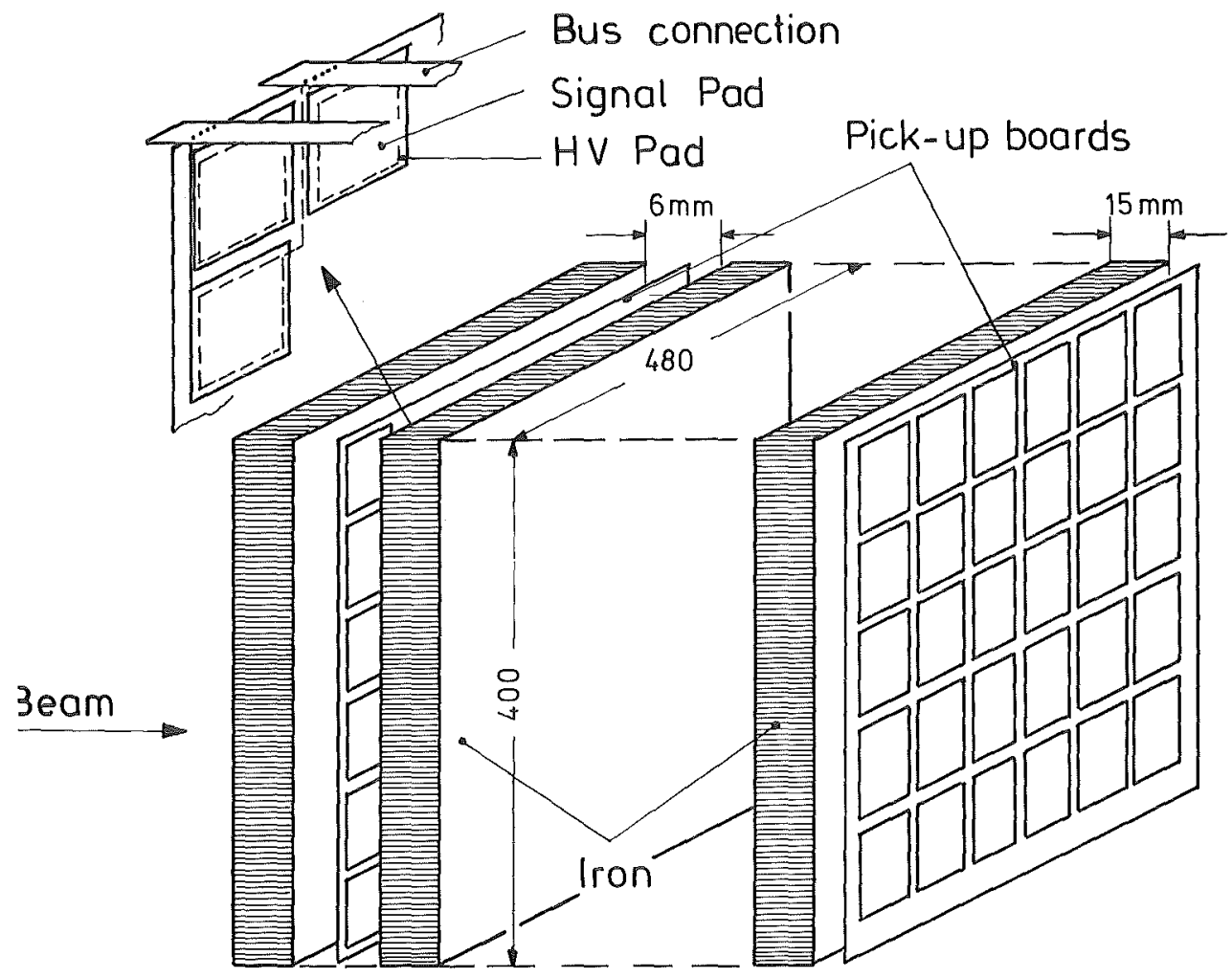

Fig. 1: Schematic view of the calorimeter set-up.

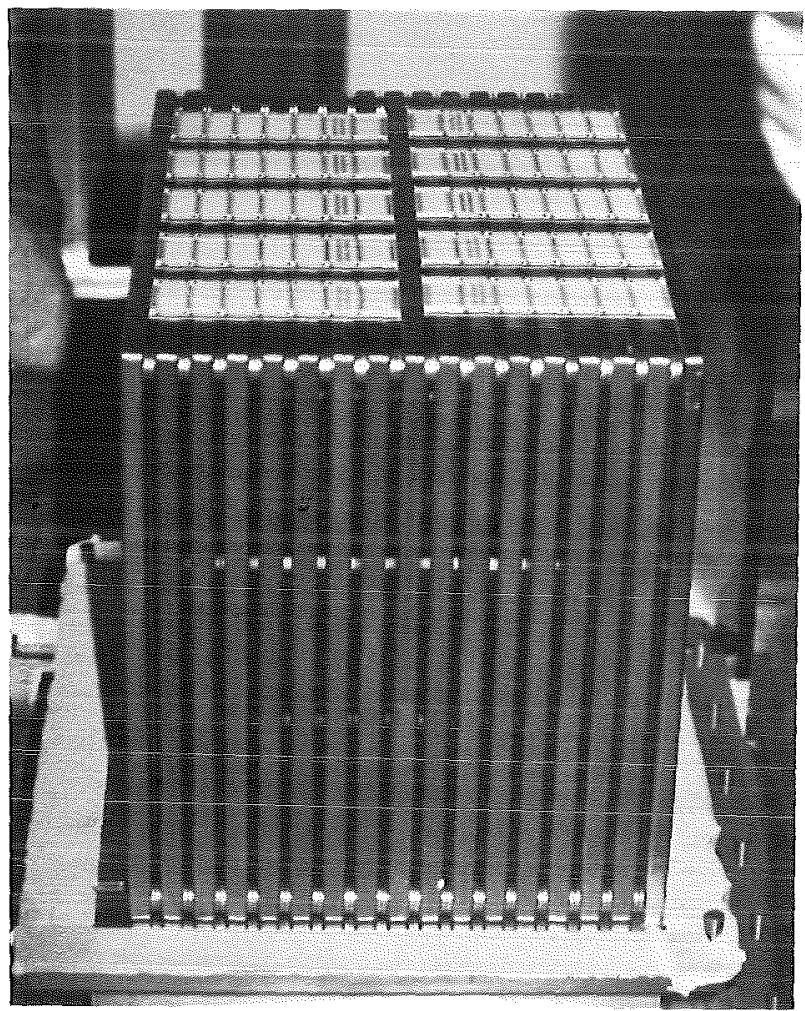

Fig. 2: Photograph of the stacking of iron slabs interspersed with pickup foils. 
The containing vessel has to be filled almost completely. We therefore have connected an extender vessel of 2 litres to the module to allow for thermal expansion of the liquid. Filling was realized through a valve on the top, and emptying through a valve at the bottom.

The module was carefully assembled, however not in a special dust-clean room. Pumping was performed for several days until a vacuum of $10^{-6} \mathrm{mb}$ had been reached. The final outgasging rate (as measured by pressure rise) was $5 \times 10^{-6} \mathrm{mb} \cdot \mathrm{l} / \mathrm{s}$. The He leak rate was determined to be less than $3 \times 10^{-9} \mathrm{mb} \cdot \ell / \mathrm{s}$.

The calorimeter was then flushed two times with raw TMS as delivered by the factory ${ }^{4}$ with a guaranteed purity of $99.9 \%$. During the second flush, we applied 2000 Watt ultrasonic power to the module and emptied it quickly within less than a minute. After being filled with purified TMS, the module was pressurized with clean argon gas to 1.4 bar abs., in order to prevent air leaking in.

\section{Purification System}

For filling, the module needs about 35 litres TMS. The liquid is purified by a system similar to that used earlier ${ }^{3}$, but in an enlarged version. The principles are sketched in Fig. 3. All the vessels are constructed from stainless steel, as are the valves and transfer lines except for the vacuum part. Vessels which could be heated accidentally have a rupture disk of 3 bar leading to the outside atmosphere.

In a first step, the liquid as it is received from factory is degassed. This is done by pumping at the condenser which is uniformly cooled by $\mathrm{CO}_{2}$ or liquid nitrogen. In a glass $\mathrm{CO}_{2}$ ice trap we check whether too much TMS is removed by strong pumping. 


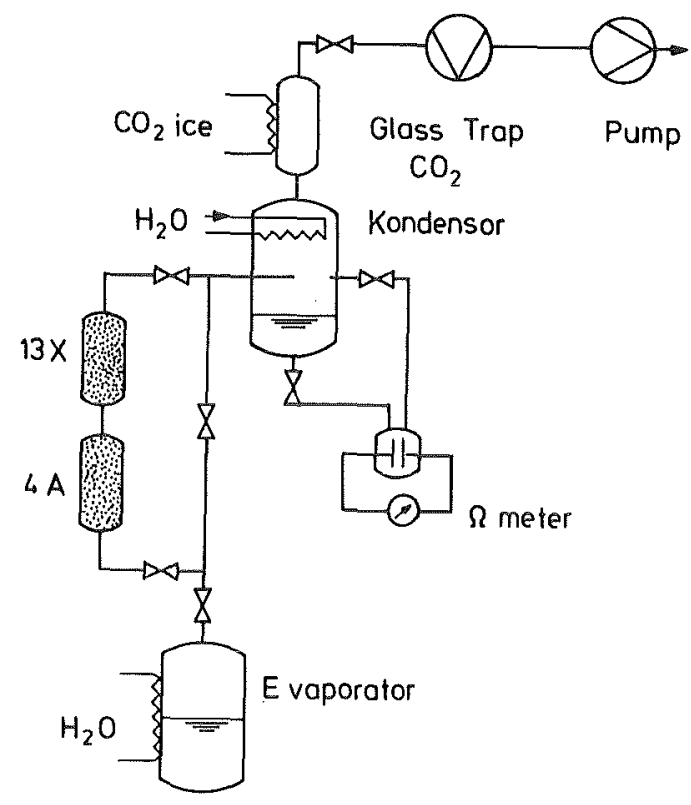

Fig. 3: The principles of the purification system.

After degassing, the liquid is distilled carefully into the upper condenser with a temperature difference of approximately $3^{0} \mathrm{C}$. The residues left in the evaporator are removed, and the distillation process repeated until no more residues are found. Typically, we observe for the first two distillation processes oily droplets or films at the botton of the evaporaton vessel.

After passing this stage, the liquid is not yet pure enough to observe electron pulses. It has to be passed over molecular sieves. We have chosen the standard combination of $4 \AA$ and $13 \AA$ zeolites. In the first sieve, water and $0_{2}$, with $2.6 \AA$ and $2.9 \AA$ diameter respectively, are effectively removed because the sieve is not loaded with TMS itself. Due to its large diameter of more than $4 \AA$, the TMS molecule can not enter the sieve granules.

Larger impurity molecules are removed in the second sieve. It is loaded with TMS too, but retains nearly its full absorption ability for polar molecules. Typically, the liquid is passed over the sieves two times with a velocity of a few times the sieve volume per second. 
The sieves have to be carefully prepared by baking them out at $350-400{ }^{\circ} \mathrm{C}$ until a pressure of $\left\langle 10^{-5} \mathrm{mb}\right.$ in warm condition is reached. When baked out, the typical pressure rise at $350^{\circ} \mathrm{C}$ is $10^{-4} \mathrm{mb} / \mathrm{s}$.

The progress of purification can conveniently be controlled by monitoring the volume resistivity of the liquid. The resistivity starts with 1012 $\Omega \cdot \mathrm{cm}$ and rises to $10^{15} 8 \cdot \mathrm{cm}$ after passing over the molecular sieves.

\section{Electroncis}

The electronic chain is shown schematically in Fig. 4a. In its principles, it is the chain as used in the CELLO LAr calorimeter ${ }^{5}$. The low noise preamplifier has been recently developed in thick film hybrid technique. Its detailed scheme is given in Fig. 4b. The amplifier was coupled directly to the detector, i.e. without using a transformer or protection resistor. The input FET used was Toshiba 2 SK 371 (equivalent to 2 SK 147). On the preamplifier card the first filter which follows the charge integration stage was also integrated. In this way, the signals on the transmission line were already reasonably large pulses, and less susceptible to pick-up noise.

The transmission line was terminated by a differential amplifier and the second filter stage. Bipolar $(\mathrm{CR})^{2}-(\mathrm{RC})^{4}$ shaping with a time constant of $1 \mu$ s was used. The signal was then digitized in a parallel conversion mode in a sample-and-hold-circuit and read out via CAMAC into an LSI-11.

A tower structure in the detector has a capacitance of $900 \mathrm{pF}$ per channel and, with this load capacitance, the mean rms noise was $0.54 \mathrm{fC}$. The variation among the 60 channels was $\sigma$ (noise) $=0.16 \mathrm{fC}$. For most of the channels, the noise did not increase when the high voltage was applied. Nevertheless, a few showed an increase in noise, which started approximately at $2 \mathrm{kV}$. From experience with several fillings of the module and from experience with other chambers, we attribute this to fine dielectric 


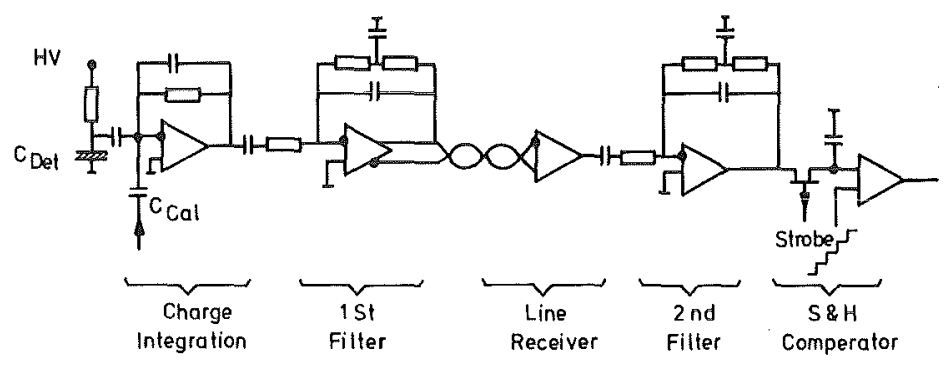

a)

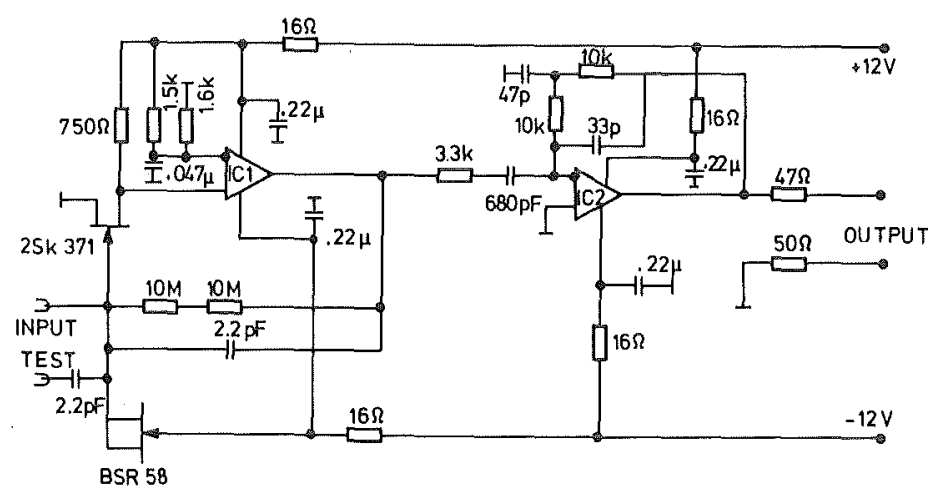

b)

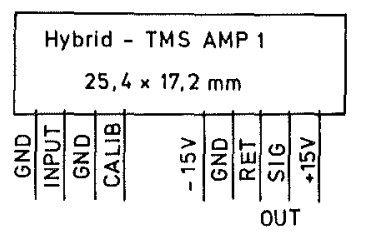

c)

Fig. 4: a) Block diagram of the electronic chain,

b) the preamplifier circuit diagram,

c) pin connections.

dust particles in the liquid. The particles seem to be suspended and are probably relics of the molecular sieves. They can be efficiently removed with a $0.5 \mu \mathrm{m}$ steel filter.

We observed break-down in one or two channels, probably due to broken connections on the polyimid foils and we therefore were restricted to $3 \mathrm{kV}$.

The preamplifiers were located directly on the steel cover and shielded by a copper box. The high voltage was filtered immediately before entering the calorimeter. The corresponding $R C$ filter was housed in a separate copper box. 
Due to the efficient shielding, we observed practically no pick-up noise, even in the experimental hall near the accelerator. The trigger rate per tower followed the expected Gaussian fall-off ${ }^{6}$ of shot noise in the preamplifier as shown in Fig. 5. It gave 1 spurious trigger per second at a threshold of $220 \mathrm{MeV}$. At higher threshold the trigger rate exceeded the amplifier noise expectation due to cosmic ray and beam background in the experimental hall.

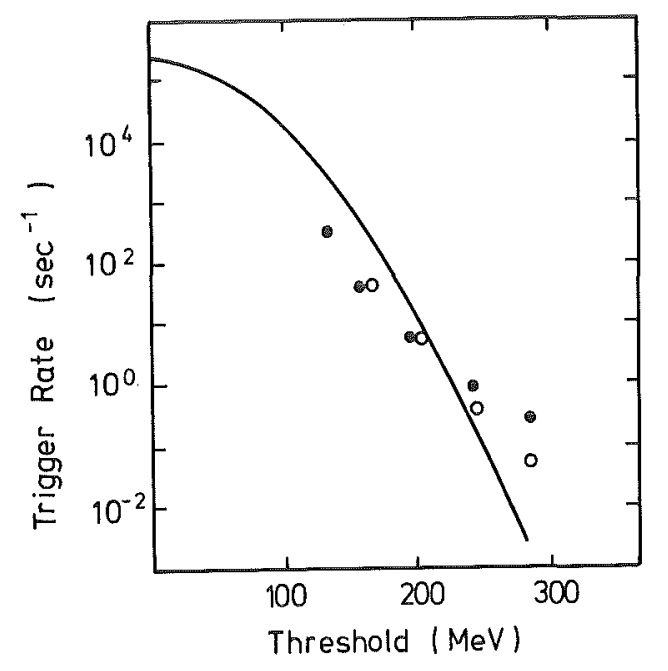

Fig. 5:

Trigger rate per tower versus energy threshold. The curve represents the expected rate due to the amplifier noise for the bipolar shaping with a time constant of $1 \mu \mathrm{s}$.

\section{Results}

We have made two performance tests with electrons of 0.6 to $5 \mathrm{GeV}$ in a test beam at DESY. The two periods were separated by 5 weeks. During the break the calorimeter was transported back to Karlsruhe, where it was stored without being kept under high voltage.

The charge yield with respect to the voltage applied is shown in Fig. 6 for $4 \mathrm{GeV}$ electrons. The yield is given as its usual value $\mathrm{G}$ which is the number of electrons released by $100 \mathrm{eV}$ deposited energy. The energy deposited in the TMS gaps has been calculated using the EGS 4 Monte Carlo code ${ }^{7}$. 


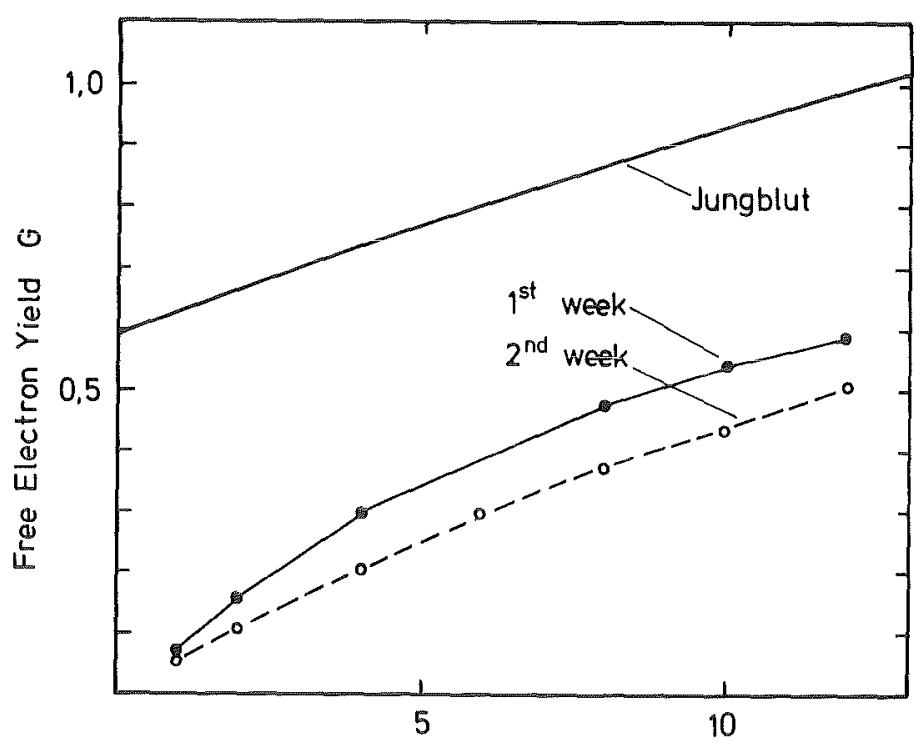

High Voltage $(\mathrm{kV} / \mathrm{cm})$
Fig. 6:

The free electron yield versus the applied high voltage. As a reference the full line shows the yield as obtained by Jungblut, ref。8.

In the figure, the yields are given separately for the 1 st and 2nd week. For comparison, the full line indicates the yield in a very clean and small test sample of Jungblut ${ }^{8}$. We observe a loss in pulse height that, assuming an exponential decay, has a decay time of 166 days or approximately half a year. We attribute this loss to impurities which are outgassing products from the polyimid foils and are led to this conclusion by the following observation:

When taking the electron yield as measured by Jungblut as a reference for an ideally pure liquid, we can calculate the lifetime of the free electrons before they are attached to impurity molecules and therefore disappear as negative ions. The result is shown in Fig. 7, in which the reciprocal lifetime is plotted with respect to the electric field. We observe that the probability for attachment increases when the electron gains energy in the electric field. This behavior sheds some light on the nature of the impurity. For instance, in case of an $\mathrm{O}_{2}$ contamination a decreasing attachment probability is expected. A rising cross-section is known, e.g., for nitrous oxides ${ }^{7}$. These oxides or similar compounds are probably outgassing products of the pick-up foils. Compounds like HCONH 2 and HCNO have been found in a mass spectrograph analysis. 


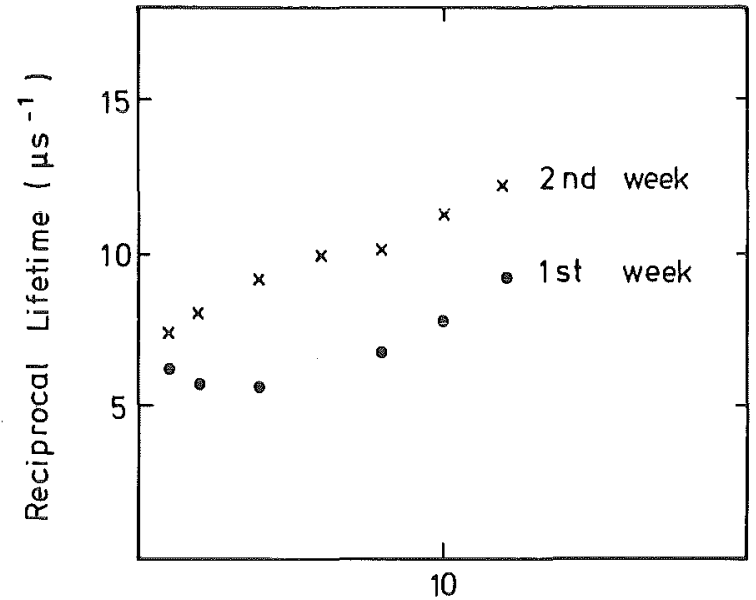

High Voltage $(\mathrm{kV} / \mathrm{cm})$
Fig. 7 :

The reciprocal lifetime of free electrons in the $1 i-$ quid versus the applied high voltage.

A remedy to avoid pulse height losses is to keep the ionization chamber always under high voltage. In our three-litre test chamber, a stack of 10 polyimid foils resembling very much the calorimeter tower size has been tested, and no signal losses has been encountered as long as a voltage of $15 \mathrm{kV} / \mathrm{cm}$ was applied. The tests had to be performed with cosmic muons and are therefore rather inaccurate. However, a loss of $5 \%$ in 10 days can be excluded.

A further remedy would be to deploy activated molecular sieves at the bottom of the module to ensure continuous cleaning.

The energy response for five different electron energies is shown in Fig. 8. The beam was centered on one tower, and in the analysis, 6 channels are summed up. These are the 4 adjacent channels and the central tower in the back plane. The values exhibit a good linearity. We have calibrated a couple of channels with beam and have checked the calibration independently from charge injection. For the latter, two different sets of calibration capacitors were used. The energy calibration coincides with the charge calibration within an rns value of $1 \%$. The differences between the two methods can be explained by signal loss due to cross talk which varied from channel to channel due to different geometrical position of signal lines. 


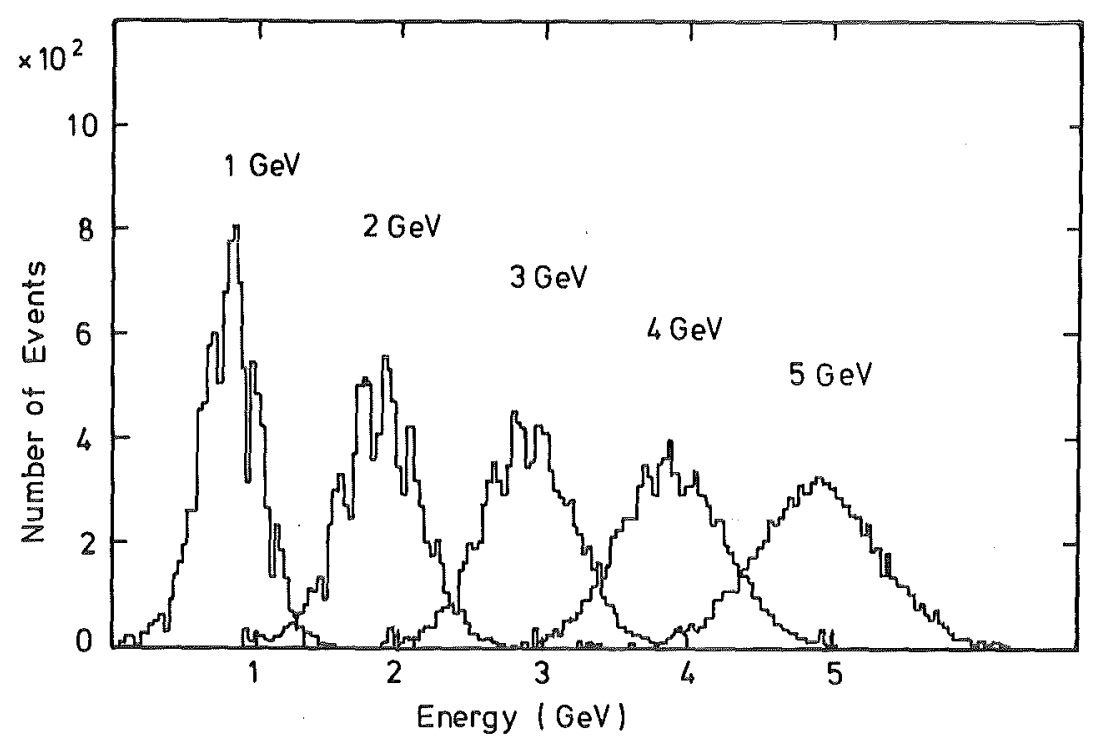

Fig. 8: Energy distribution for incoming electrons of $1-6 \mathrm{GeV}$ hitting the centre of a tower.

The energy resolution is shown in Fig. 9. It follows the $1 / \sqrt{\mathrm{E}}$ law. The black points are mean values for the beam centered on pads in different regions in the calorimeter. They agree well with predicted figures of the EGS 4 code, which gives the horizontal straight line as mean value.

We have measured down to $0.6 \mathrm{GeV}$ for one particular channel. The outcome is presented as open circles in the figure. We observe that at lower energies the resolution deteriorates due to electronic noise, which is no longer negligable. The dashed line is a fit to the data points which yields a noise contribution of $138 \mathrm{MeV}$. A distribution of noise figures of all the channels in the calorimeter is shown in Fig. 10. The mean corresponds to $53 \mathrm{MeV}$ shower energy. We therefore expect $130 \mathrm{MeV}$ equivalent noise when adding up 6 towers, which coincides well with the above value. In Fig. 10, three channels show up with larger noise that is related to high voltage and probably caused by dielectric dust particles in the liquid as mentioned in section 4. When we include these channels, the total noise in the calorimeter corresponds to $225 \mathrm{MeV}$ shower energy. 


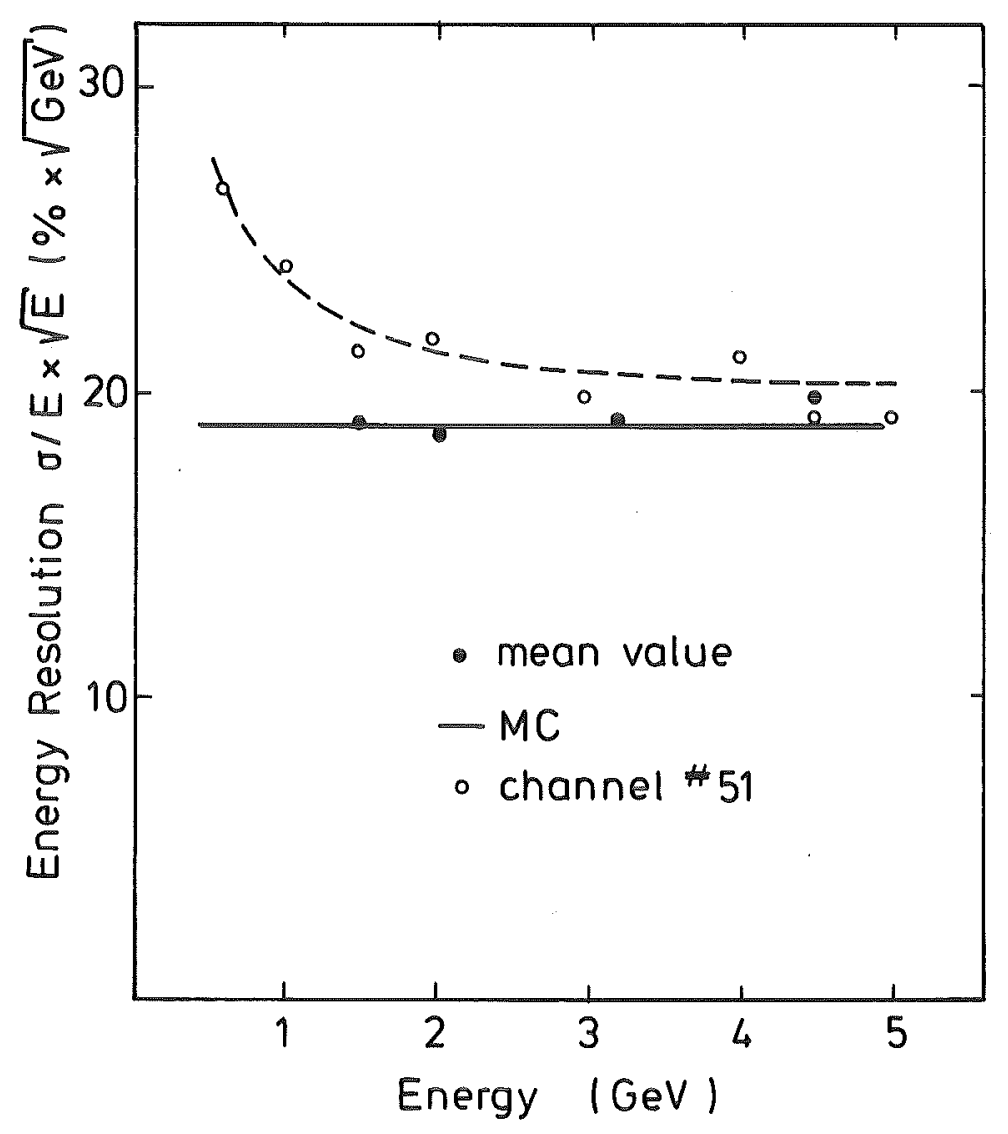

Fig. 9:

Normalized

energy resolution versus the incoming electron energy. The horizontal line represents the mean value of a Monte Carlo calculation using the EGS 4 code. The black dots are mean values of channels in different parts of the calorimeter. The open circles are values for one particular channel in the

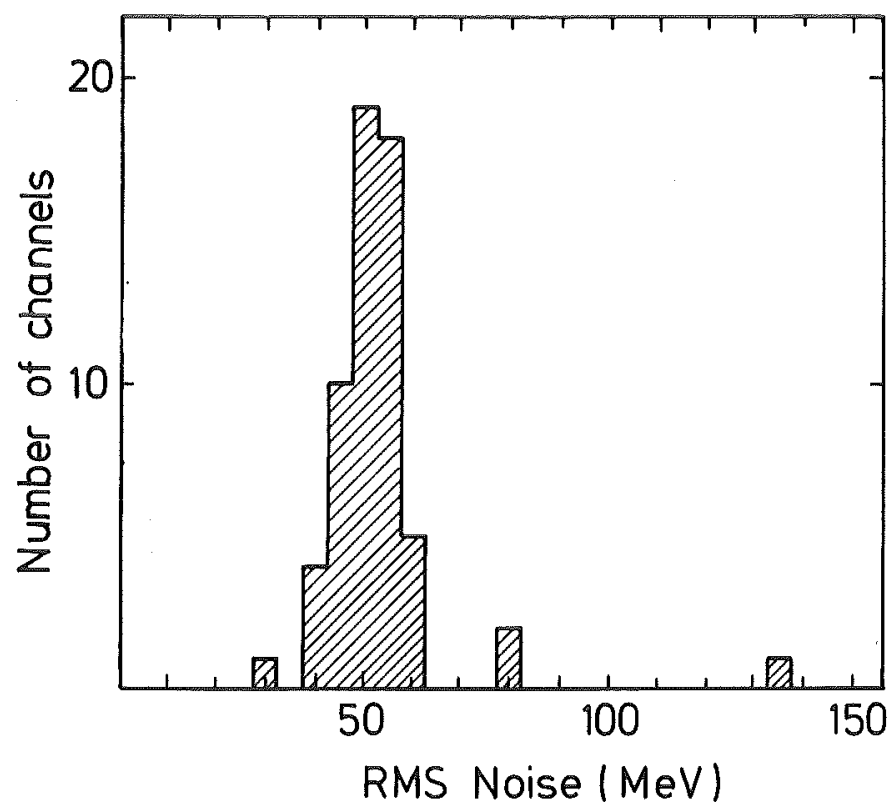

Fig. 10:

The energy equivalent noise of all channels in the calorimeter. 


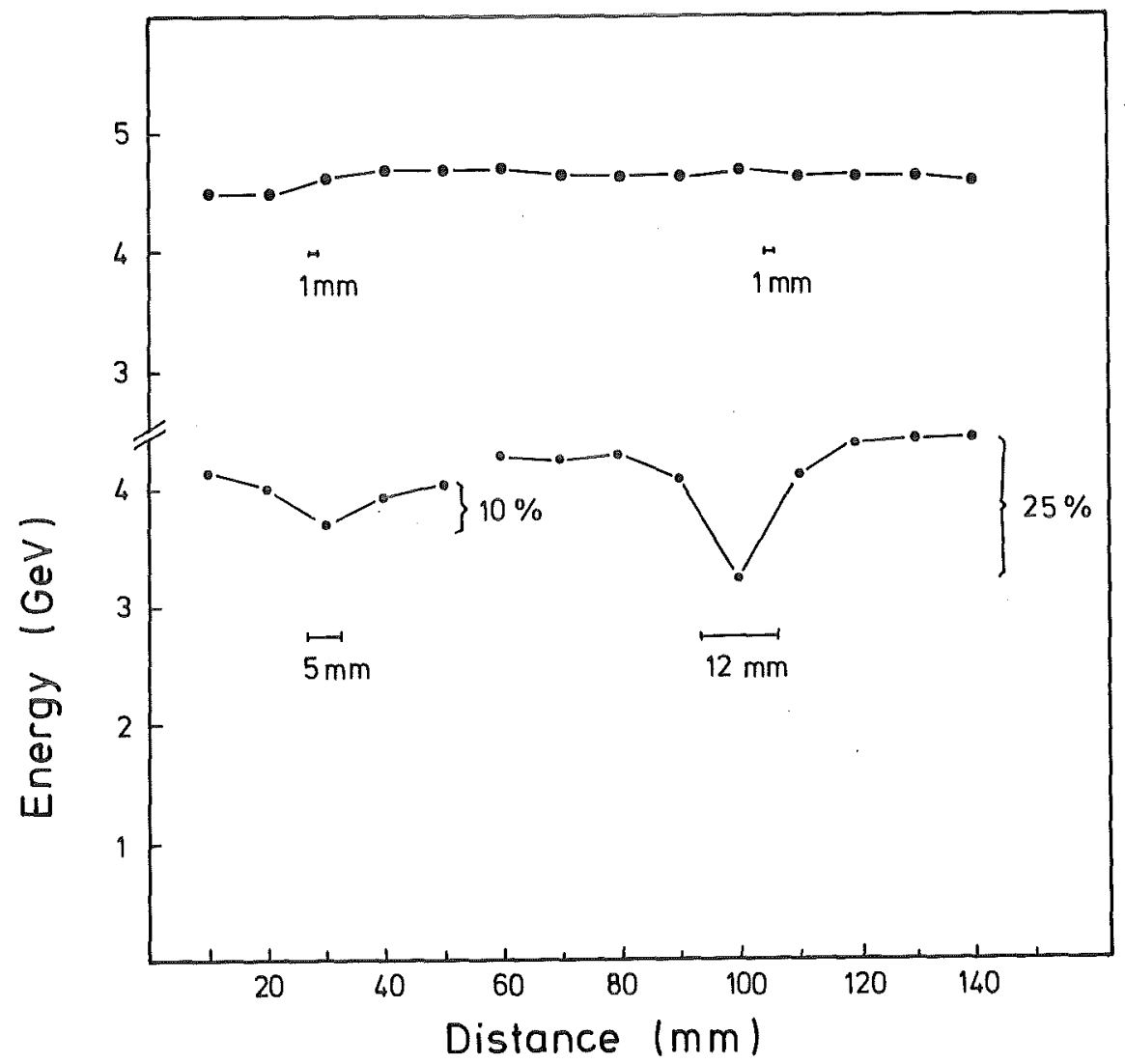

Fig. 11: Energy versus position of beam incidence. Shown are scans over a pad separation of $1 \mathrm{~mm}$ and $5 \mathrm{~mm}$ and over a washer of $12 \mathrm{~mm}$ diameter.

We have checked the homogeneity of charge collection in the calorimeter. The results are given in Fig. 11. Plotted is the energy measured when the module is moved through the beam on a scanning table. The usual pad separation is $1 \mathrm{~mm}$, and for this distance no charge loss can be observed as is demonstrated by the upper curve. The position of pad boundaries are marked below the measured points. On the other hand, a pad separation of $5 \mathrm{~mm}$ already entails a signal loss of 10\%. Such a distance between the pads was necessary where several connection lines of inner pads passed through. Also shown is the signal loss when the beam hits the centre of a washer, which corresponds to $25 \%$. However, only $4 \%$ of the calorimeter surface is obstructed by these washers. 


\section{Conelusions}

We have operated for the first time a calorimeter module with a room temperature liquid. Purification by distillation over molecular sieves is straight forward, but has to be performed carefully. For a reasonably high charge output, molecular liquids need high voltages. We found that care for dust free chambers is necessary. The operation itself causes no major problems, and in spite of having occasional voltage breakthrough, the signals had a lifetime of half a year, even when the calorimeter was not kept under high voltage. Electronically, the module was very stable to operate due to the efficient shielding of the preamplifiers, and a low trigger thresholds of $200 \mathrm{MeV}$ with 1 spurious trigger per second was achieved.

We wish to express our appreciation to the skillful help of R. Dittmann in setting up the calorimeter module and the purification system and $C$. Miller for help during data taking. We would like to thank Profs. G. Flugge and $B$. Zeitnitz for continuous interest and encouraging support and $\mathrm{Dr}$. B. Gunderson for reading the manuscript. We are indebted for the beam to the DESY operations group. This work was partly supported by the Bundesministerium für Forschung und Technologie, Bonn, Germany. 


\section{References}

(1) See e.g.

C.W. Fabjan and T. Ludlam, Ann.Rev.Nucl.Part.Sci. 32 (1982) 335

J. Engler, Nucl.Ingtr. and Meth. A235 (1985) 301

(2) T.A. Gabriel et al., IEEE Trans.Nucl.Sci.NS-32 (1985) 697 M. de Vincenzi et al., CERN Preprint EP/86-12, submitted to Nuc1. Instr. and Meth.

(3) J. Engler and H. Keim, Nucl.Instr, and Meth. 223 (1984) 47 R.A. Holroyd and D.F. Anderson, Nucl.Instr. and Meth. A236 (1985) 294

(4) Alfa Products, represented in Europe by Ventron GmbH, Karlsruhe

(5) H.J. Behrend et al., Physica Scripta 23 (1981) 610

(6) D. Wolf and H. Brehm, Archiv der Elektrischen Übertragung 27 (1973) 477

(7) R.L. Ford and W.R. Nelson, EGS Code, SLAC Rep. 210 (1978)

(8) H. Jungblut, Diploma work at the Freie Universität Berlin (1975), not published

(9) A. Henglein, Can.J.Chem. 55 (1977) 2112 